\title{
Seeing Stars
}

\section{Universality in the Evolution of Orientation Columns in the Visual Cortex Matthias Kaschube, Michael Schnabel, Siegrid Löwel, David M. Coppola, Leonard E. White, and Fred Wolf \\ Science 1194869 Published online 4 November 2010 [DOI:10.1126/science.1194869]}

\section{Recommended with a Commentary by Elisha Moses, Weizmann Institute}

One of the deep questions that drive the Physics of Biology is: to what extent do general physical principles govern biological organisms? While cells must obviously obey the rules of Physics, they are notorious 'control freaks', in the sense that biology maintains strict control over the activation of genes and the timely production of proteins. The brain is a wonderful candidate for searching for unified underlying principles, because information capacity in the genes is insufficient to code for so much structural information. However, to date there is still very little evidence of such principles in action.

Such a system has now been identified by Kaschube et al. [1], who examined the visual cortex of three small mammalian animals (galago, ferret and tree shrew), which belong to very disparate genealogical families that were separated more than 65 million years. In the primary visual cortex (V1), neurons are selective to the particular orientation of a stimulus (e.g. a bar that is moving either vertical or horizontal). In the species that were examined (but not, for example, in rodents) the preferred orientation changes smoothly along the cortical surface and extends into the cortex to form 'orientation columns'. The vector fields on the surface must have singularities where areas of different orientation preference meet (like combing a hedgehog), which are called 'pinwheels'.

Kaschube et al. first measured the number of pinwheels per unit area, normalized by the orientation column size, and showed that it is constant in the animals observed (the constant turns out to be $\pi$ ). This universal behavior develops even if they are reared in darkness, which otherwise impairs their sight. Not only the average value is constant, but the statistics of the standard deviation and of the nearest neighbor correlations were shown to be the same in all three animal species.

To explain these similarities, the model proposed involves a complex order parameter describing the local orientation preference (direction and amplitude). The dynamics involve both a local, linear Swift-Hohenberg term and a long range, nonlinear third order term which is invariant under the simple symmetries of rotation, translation, phase shift and permutation. According to the authors, any model that preserves these symmetries will give the same statistics for the pinwheels.

The success of this approach is important and significant not only in solving the long standing problem of how the map of pinwheels is determined, but also in pointing at a complex Biological system where simple underlying and unifying Physical principles can be found. 